\title{
Overexpression of long noncoding RNA CUDR promotes hepatic differentiation of human umbilical cord mesenchymal stem cells
}

\author{
YABIN YU ${ }^{1}$, MENG $\mathrm{LI}^{2}$, YAN SONG $^{1}$, JIANBO XU $^{1}$ and FUZHEN QI ${ }^{1}$ \\ ${ }^{1}$ Department of Hepatobiliary Surgery, The Affiliated Huaian No. 1 People's Hospital of Nanjing Medical University, \\ Huaian, Jiangsu 223300; ${ }^{2}$ Department of Pathology, Xuzhou Medical University, Xuzhou, Jiangsu 221004, P.R. China
}

Received June 1, 2019; Accepted October 23, 2019

DOI: $10.3892 / \mathrm{mmr} .2019 .10897$

\begin{abstract}
Previous studies have shown that long noncoding RNAs (lncRNAs) are capable of regulating cell differentiation and pluripotency. The objective of the present study was to explore the effect of IncRNA cancer upregulated drug resistant (CUDR) on the hepatic differentiation of human umbilical cord mesenchymal stem cells (HuMSCs). HuMSCs were subjected to a hepatogenic differentiation protocol. The level of CUDR was monitored by reverse transcription-quantitative PCR (RT-qPCR) following certain stages of hepatic differentiation. Lentivirus transfection was used to achieve CUDR overexpression. The hepatocyte-related proteins and mRNAs were then examined by immunofluorescence, ELISA and RT-qPCR analyses. The results showed that CUDR was upregulated during the hepatic differentiation of HuMSCs. Upregulation of CUDR can improve hepatic differentiation of HuMSCs, including hepatocyte-related genes and proteins. In addition, it was also found that liver-enriched transcription factors were upregulated after CUDR overexpression. Moreover, there was an association between the Wnt/ $\beta$-catenin pathway and CUDR. In summary, these results demonstrated that the overexpression of CUDR could improve the hepatic differentiation of HuMSCs, therefore it could be an ideal source for regenerative therapy.
\end{abstract}

\section{Introduction}

Liver dysfunction is a global health issue and currently the only successful treatment for end-stage liver disease is liver transplantation (1). However, due to donor shortage and immunological rejection, its clinical application is limited (2). To this end, bio-artificial liver support systems and hepatocyte transplantation are two potential complementary therapies

Correspondence to: Dr Fuzhen Qi, Department of Hepatobiliary Surgery, The Affiliated Huaian No. 1 People's Hospital of Nanjing Medical University, 1 West Huanghe Road, Huaian, Jiangsu 223300, P.R. China

E-mail: qifuzhen2017@163.com

Key words: long noncoding RNA, mesenchymal stem cell, differentiation, hepatocyte, liver failure for patients with end-stage liver disease. Developments in stem cells, particularly mesenchymal stem cells (MSCs), have highlighted a new source of liver cells as they have the ability to differentiate into hepatocyte-like cells via the addition of cytokines in vitro (3).

MSCs can be isolated from various body tissues, including amniotic fluid, umbilical cord placenta, bone marrow and adipose tissue (4,5). Human umbilical cord (Hu)MSCs are recognized as an ideal supply for cell therapy because of their low immunogenicity, abundant source and freedom from ethical issues (6). Our recent study showed the efficacy of HuMSCs in regenerative medicine and that HuMSCs hold numerous advantages over bone marrow-derived MSCs (BMSCs), including higher potential for proliferation and differentiation abilities in vitro (7). However, the efficacy of hepatic differentiation of MSCs is still insufficient for clinical application (8). Therefore, it is necessary to find a new differentiation method to achieve a higher efficient transdifferentiation.

Long noncoding RNAs (lncRNAs) are a class of RNAs $>200$ nucleotides in length that cannot encode proteins. It has recently been reported that some IncRNAs can play important roles in cellular activities, including cell proliferation, self-renewal, differentiation and apoptosis $(9,10)$. For example, HOTAIR improves MSC differentiation and is associated with senescence-associated DNA methylation (11).

Studies on lncRNA cancer upregulated drug resistant (CUDR) have mainly focused on cancer cells and other related molecular mechanisms. It is a novel noncoding RNA gene, which was found to influence the proliferation, apoptosis and cell cycle progression of colorectal cancer cells (12). Moreover, CUDR has the ability to promote liver cancer growth and hepatocyte-like stem cell malignant transformation epigenetically by cooperating with set domain-containing $1 \mathrm{~A}$, histone lysine methyltransferase (13). Little is known regarding the expression of CUDR in hepatocytes or in the differentiation of hepatocytes. A previous study has highlighted the role of CUDR in embryo stem cell growth and hepatic differentiation (14). However, the function of CUDR in the hepatic differentiation of MSCs remains unclear. The present study demonstrated that expression of CUDR significantly increased during the hepatic differentiation of HuMSCs, and that it promoted hepatic differentiation. Moreover, these results showed that CUDR not only regulated liver-enriched factors, but also inhibited the Wnt/ $\beta$-catenin pathway. 


\section{Materials and methods}

Culture and differentiation of HuMSCs. HuMSCs were purchased from Beijing Beina Chuanglian Biotechnology Institute. Cells were cultured in $25-\mathrm{cm}^{2}$ culture flasks containing HyClone ${ }^{\mathrm{TM}}$ Dulbecco's modified Eagle's medium (HyClone; GE Healthcare Life Sciences), supplemented with $10 \%$ fetal bovine serum (Gibco; Thermo Fisher Scientific, Inc.), $100 \mathrm{U} / \mathrm{ml}$ penicillin and $100 \mu \mathrm{g} / \mathrm{ml}$ streptomycin (Gibco; Thermo Fisher Scientific, Inc.). Cells were grown at $37^{\circ} \mathrm{C}$ under $5 \% \mathrm{CO}_{2}$ atmosphere. The culture medium was replaced every 3 days and the HuMSCs were digested with trypsin (Gibco; Thermo Fisher Scientific, Inc.) once they reached $70-80 \%$ confluency. The cells in the fourth passage were used for further differentiation. Before hepatic differentiation, the multipotency of the cultured HuMSCs was confirmed by differentiation experiments. The cells were treated with osteogenic medium containing L-glutamine, decamethasone, ascorbate and $\beta$-glycerophosphate (Sigma-Aldrich; Merck KGaA) and chondrogenic medium containing h-Insulin, L-glutamin, dexamethasone, indomethacin and 3-isobuty-I-methyl-xanthine (Sigma-Aldrich; Merck KGaA) according to previous studies $(15,16)$.

Alizarin red staining. Cells were washed by PBS twice and fixed in $10 \%$ paraformaldehyde for at $4^{\circ} \mathrm{C}$ for $10 \mathrm{~min}$. Alizarin red $(0.1 \%)$ was added at $37^{\circ} \mathrm{C}$ for $30 \mathrm{~min}$. Following washing in distilled water, they were observed under an inverted microscope (magnification, x100).

Type II collagen staining. Cells were fixed with $4 \%$ paraformaldehyde at $4^{\circ} \mathrm{C}$ for $30 \mathrm{~min}$, permeabilized with $2 \%$ Triton X-100 and labeled with monoclonal antibody anti-type II collagen antibody (SC-52658, Santa Cruz Biotechnology, Inc.). They were observed under an inverted microscope (magnification, $\mathrm{x} 100)$.

Hepatic differentiation. To induce hepatic differentiation, the growth medium was replaced with differentiation medium described below when cells in passage four reached $80 \%$ confluency, as based on a previous protocol (3). Differentiation was induced by treating MSCs with liver-specific growth factors: Days 0-2, Iscove's modified Dulbecco's medium (IMDM, Gibco; Thermo Fisher Scientific, Inc.) with $20 \mathrm{ng} / \mathrm{ml}$ epidermal growth factor (PeproTech, Inc.) and $10 \mathrm{ng} / \mathrm{ml}$ basic fibroblast growth factor (bFGF; PeproTech, Inc.); days 3-9, IMDM supplemented with $20 \mathrm{ng} / \mathrm{ml}$ hepatocyte growth factor (PeproTech, Inc.), $10 \mathrm{ng} / \mathrm{ml}$ bFGF and $0.61 \mathrm{~g} / \mathrm{ml}$ nicotinamide (Sigma-Aldrich; Merck KGaA); from day 9 onwards, IMDM containing $20 \mathrm{ng} / \mathrm{ml}$ oncostatin M (PeproTech, Inc.), $1 \mu \mathrm{mol} / 1$ dexamethasone (Sigma-Aldrich; Merck KGaA) and $50 \mathrm{mg} / \mathrm{ml}$ insulin/transferring/selenium (Sigma-Aldrich; Merck KGaA). The hepatic differentiation medium was replaced every 3 days. The progression of differentiation from HuMSCs to hepatocytes (at days 14 and 28) was analyzed.

Immunocytochemistry analysis. After induction, cells were fixed with $4 \%$ paraformaldehyde for $30 \mathrm{~min}$ (Beyotime Institute of Biotechnology) and blocked in 10\% BSA (Gibco; Thermo Fisher Scientific, Inc.) at $37^{\circ} \mathrm{C}$ for $30 \mathrm{~min}$. Slides were then incubated with primary antibodies (ALB sc-271605, AFP sc-8399, CYP3A4 sc-365415 and $\beta$-catenin sc-47724, Santa Cruz Biotechnology, Inc.), including mouse monoclonal anti-albumin (ALB; 1:200), mouse monoclonal anti- $\alpha$ fetoprotein (AFP; 1:200), mouse monoclonal anti-cytochrome P450 $3 \mathrm{~A} 4$ (CYP3A4; $1: 200)$ and mouse monoclonal anti- $\beta$-catenin $(1: 200)$ overnight at $4^{\circ} \mathrm{C}$. After washing, cells were incubated with FITC-conjugated (Dylight 594 and Alexa 488) goat anti-mouse immunoglobulin $\mathrm{G}$ secondary antibody (1:1,000, sc-516140; Santa Cruz Biotechnology, Inc.) at $37^{\circ} \mathrm{C}$ for $45 \mathrm{~min}$. After rinsing, the nuclei were stained with DAPI (Sigma-Aldrich; Merck KGaA) at $37^{\circ} \mathrm{C}$ for 5 min and cells were then observed with a fluorescence microscope (Olympus Corporation) at $\mathrm{x} 200$ magnification.

Reverse transcription-quantitative PCR (RT-qPCR). Total RNA from cells was isolated using TRIzol reagent (Invitrogen; Thermo Fisher Scientific, Inc.) according to the manufacturer's protocol. The cDNA was synthesized using PrimeScript ${ }^{\mathrm{TM}} \mathrm{RT}$ reagent kit (Takara Biotechnology Co., Ltd.) in a $20 \mu \mathrm{l}$ reaction containing 100-200 ng of total RNA. All samples were reacted at $37^{\circ} \mathrm{C}$ for $60 \mathrm{~min}$, heated at $95^{\circ} \mathrm{C}$ for $5 \mathrm{~min}$, and finally held at $4^{\circ} \mathrm{C}$. The products were then quantified via RT-qPCR using SYBR Green Master Mix (Takara Biotechnology Co., Ltd.) with the primer sequences listed in Table I. The analysis of the melting curve was achieved to exclude nonspecific amplification products (16). Human GAPDH was used as an internal control for PCR. Thermocycling conditions were: Initial denaturation: $95^{\circ} \mathrm{C}$ for $30 \mathrm{sec}, 95^{\circ} \mathrm{C}$ for $5 \mathrm{sec}$ and $60^{\circ} \mathrm{C}$ for $34 \mathrm{sec}$ for 30 cycles. Fold changes were calculated using the relative quantification $\left(2^{-\Delta \Delta \mathrm{Cq}}\right)$ method (17).

CUDR transfection. CUDR lentiviral plasmid LV5-CUDR-homo and negative control vectors LV3-NC were purchased from Shanghai GenePharma Co., Ltd. Cells were seeded in 6 -well plates $\left(5 \times 10^{4}\right.$ cells/well $)$ and then incubated with $1 \times 10^{8} \mathrm{TU} / \mathrm{ml}$ lentivirus $(4 \mu \mathrm{l}), 5 \mu \mathrm{g} / \mathrm{ml}$ polybrene (Shanghai GenePharma Co., Ltd.) and complete medium. After transfection, lentivirus-infected HuMSCs were subjected to puromycin for selection. After 7 days of selection, the stable transfected cell line was identified, and overexpression of CUDR was confirmed by RT-qPCR analysis.

ELISA. The cell culture supernatants were collected after hepatic differentiation of HuMSCs on 6-well plates. The human ALB and blood urea nitrogen $(\mathrm{BuN})$ concentrations of culture supernatants were measured using ELISA kits (ALB, ab108788, Abcam; BuN, SKT-213-192, StressMarq Biosciences Inc.), referring to the manufacturer's protocol.

Western blotting. Cells were lysed in RIPA buffer (Invitrogen; Thermo Fisher Scientific, Inc.) and a protease inhibitor cocktail before being heated at $95^{\circ} \mathrm{C}$ for $10 \mathrm{~min}$. Then, the lysates were centrifuged at $15,000 \mathrm{x} \mathrm{g}$ for $25 \mathrm{~min}$ at $4^{\circ} \mathrm{C}$ to remove the debris. $10 \mu \mathrm{g}$ protein per lane was separated by $10 \%$ SDS-PAGE and transferred to $0.45-\mu \mathrm{m}$ PVDF membranes. Primary antibodies against hepatocyte nuclear factor $4 \alpha$ (HNF4 $\alpha$, sc-374299, Santa Cruz Biotechnology, Inc.), hepatocyte nuclear factor $3 \beta$ (HNF3 $\beta$, sc-374376, Santa Cruz Biotechnology, Inc.), hepatocyte nuclear factor 6 (HNF6, 
Table I. Sequences of reverse transcription-quantitative PCR primers.

\begin{tabular}{|c|c|c|c|}
\hline Genes & Primer sequence $\left(5^{\prime}-3^{\prime}\right)$ & Fragment length (bp) & Annealing temperature \\
\hline \multirow[t]{2}{*}{ ALB } & F: TGCTTGAATGTGCTGATGACAGGG & & \\
\hline & R: AAGGCAAGTCAGCAGGCATCTCATC & 162 & 60 \\
\hline \multirow[t]{2}{*}{ AFB } & F: GAAACCCACTGGAGATGAACAGTC & & \\
\hline & R: AAGTGGGATCGATGCAGGA & 190 & 60 \\
\hline \multirow[t]{2}{*}{ TAT } & F: TGAGCAGTCTGTCCACTGCCT & & \\
\hline & R: ATGTGAATGAGGAGGATCTGAG & 359 & 60 \\
\hline \multirow[t]{2}{*}{ G6P } & F: GCTGGAGTCCTGTCAGGCATTGC & & \\
\hline & R: TAGAGCTGAGGCGGAATGGGAG & 349 & 60 \\
\hline \multirow[t]{2}{*}{ CYP3A4 } & F: TGTGCCTGAGAACACCAGAG & & \\
\hline & R: GCAGAGGAGCCAAATCTACC & 202 & 60 \\
\hline \multirow[t]{2}{*}{$\alpha 1 \mathrm{AT}$} & F: CTGGGACAGTGAATCGACAATGC & & \\
\hline & R: TCTGTTTCTTGGCCTCTTGGTG & 560 & 54 \\
\hline \multirow[t]{2}{*}{ GAPDH } & F: AGAAGGCTGGGGCTCATTTG & & \\
\hline & R: AGGGCCATCCACAGTCTTC & 258 & 52 \\
\hline \multirow[t]{2}{*}{ HNF4 $\alpha$} & F: GCACCAACCTCAACGC & & \\
\hline & R: AGGCTGCTGTCCTCATAG & 313 & 56 \\
\hline \multirow[t]{2}{*}{ CUDR } & F: ACGCTAACTGGCACCTTGTT & & \\
\hline & R: CTCCGGACTGCTTCAAGTGT & 124 & 56 \\
\hline \multirow[t]{2}{*}{ HNF3 $\beta$} & F: GCACCTGCAGATTCTGATTTT & & \\
\hline & R: GACTTCCCTGCAACAACAGC & 66 & 60 \\
\hline \multirow[t]{2}{*}{ HNF6 } & F: CCTGGAGCAAACTCAAATCC & & \\
\hline & R: TTCTTTCCTTTTGCATGCTG & 116 & 60 \\
\hline \multirow[t]{2}{*}{$\mathrm{CEBP} \alpha$} & F: CAACACTTGTATCTGGCCTCTG & & \\
\hline & R: CGAGCAAAACCAAAACAAAAC & 112 & 60 \\
\hline \multirow[t]{2}{*}{$\beta$-catenin } & F: ATTGTCCACGCTGGATTTTC & & \\
\hline & R: AGGTCTGAGGAGCAGCTTCA & 142 & 58 \\
\hline
\end{tabular}

ALB, albumin; AFB, $\alpha$ fetoprotein; CYP3A4, cytochrome P450 3A4; HNF4 $\alpha$, hepatocyte nuclear factor $4 \alpha$; RT-qPCR, reverse transcription quantitative PCR; CUDR, cancer up-regulated drug resistant; CUDR, cancer upregulated drug resistant; HNF3 $\beta$, hepatocyte nuclear factor 3 $\beta$; HNF6, hepatocyte nuclear factor 6; CEBP $\alpha$, enhanced binding protein $\alpha$; G6P, glucose-6-phosphatase; TAT, tyrosine aminotransferase; $\alpha 1 \mathrm{AT}$, $\alpha 1$-antitrypsin.

sc-365318, Santa Cruz Biotechnology, Inc.), enhanced binding protein $\alpha(\mathrm{CEBP} \alpha, \mathrm{sc}-133239$, Santa Cruz Biotechnology, Inc.), $\beta$-catenin (sc-47724, Santa Cruz Biotechnology, Inc.) and GAPDH (all at 1:2,000 dilution) were incubated overnight at $4^{\circ} \mathrm{C}$ and washed with TBS containing $0.1 \%$ Tween-20. Secondary antibody (1:5,000; Santa Cruz Biotechnology, Inc. goat anti-mouse IgG-HRP, sc-2005) was incubated with membranes for $2 \mathrm{~h}$ at room temperature. Visualization was performed with an enhanced chemiluminescence substrate (Thermo Fisher Scientific, Inc.) and the band intensities were analyzed using Image J software (version 1.8.0, National Institutes of Health) for quantitative calculation.

Dual-luciferase reporter gene assay. Cells were first seeded into 24-well plates and co-transfected with TOP/FOP Flash plasmid and Renilla TK-Luciferase vector (Shanghai GenePharma Co., Ltd.) with Lipofectamine ${ }^{\circledR} 2000$ reagent (11668027, Invitrogen). Then, $48 \mathrm{~h}$ after transfection, cells were harvested and lysed for luciferase assay (Promega Corporation). The luciferase activity of each samples was normalized with its respective Renilla luciferase activity.
Statistical analysis. The significant differences between mean values obtained from at least three independent experiments were received. Each value was presented as the mean \pm SD. ANOVA followed by a post-hoc Tukey's test was used for comparisons between various groups, with $\mathrm{P}<0.05$ considered statistically significant. Statistical analysis was carried out using SPSS 16.0 software (SPSS, Inc.).

\section{Results}

Hepatocyte differentiation of HuMSCs in vitro. HuMSCs were successfully induced to osteoblasts and chondrocytes, as evidenced by positive staining for alizarin red and type II collagen, respectively (Fig. 1A). After treatment with hepatocyte induction media, the morphology of HuMSCs observed by phase contrast microscopy did not notably change until day 14. However, from day 15, the cells were found to modify their phenotype from a spindle to a polygonal shape, and granules in the cytoplasm also gradually accumulated (Fig. 1B). The progression of differentiation from HuMSCs to hepatocytes (at days 14 and 28) was analyzed via RT-qPCR at 
A
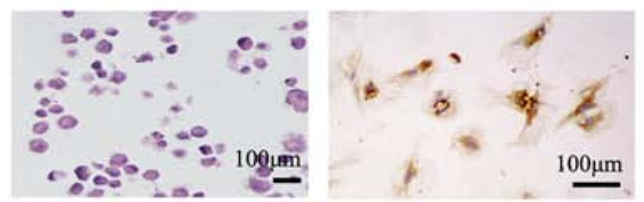

\section{$\mathrm{C}$}
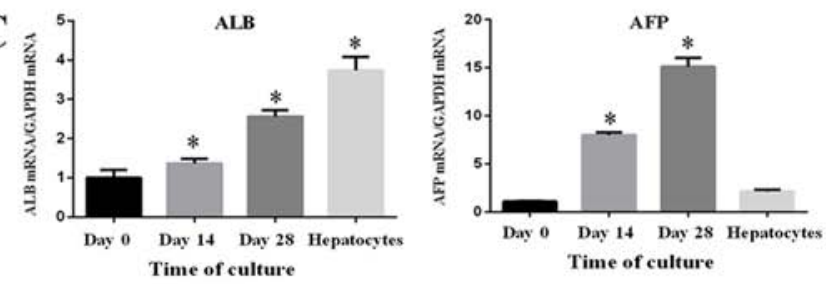

D
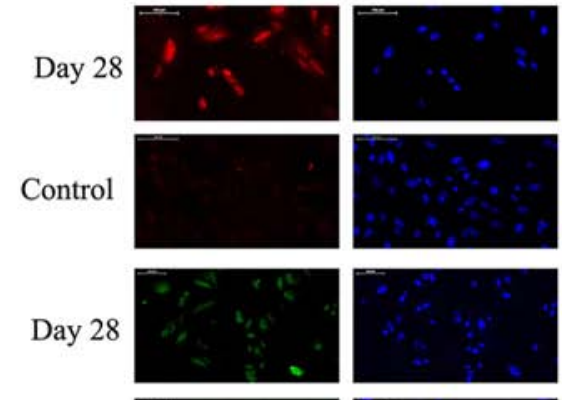

Control
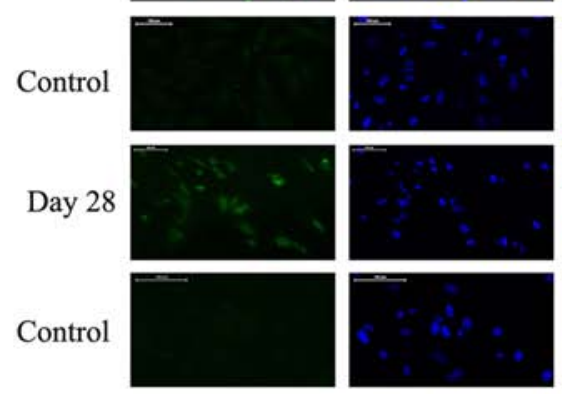

B
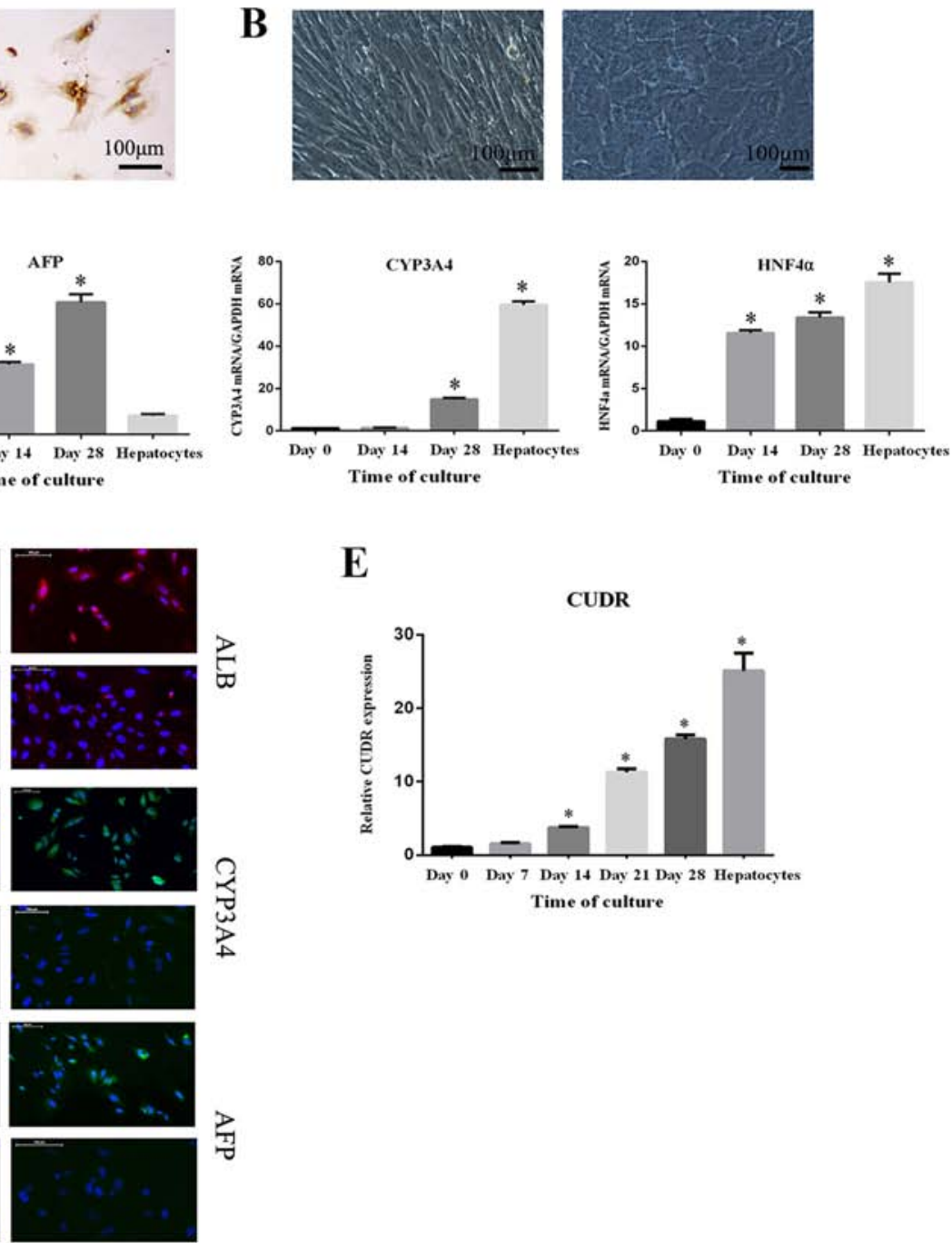

$\mathbf{E}$

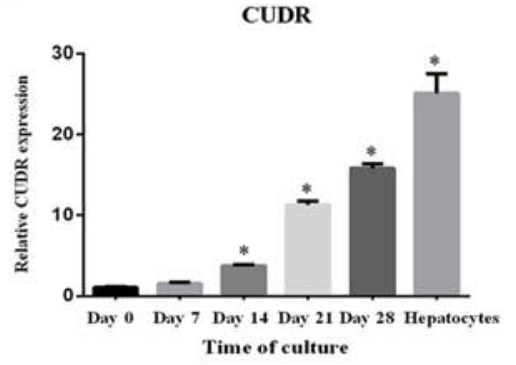

Figure 1. Hepatic differentiation of HuMSCs. (A) HuMSCs were identified by osteogenic and chondrogenic differentiation. (B) Morphological changes observed as HuMSCs differentiated into hepatocytes with the addition of cytokines. (C) mRNA levels of ALB, AFP, CYP3A4 and HNF4 $\alpha$ after differentiation were analyzed via RT-qPCR. (D) ALB, AFP, CYP3A4 protein levels after differentiation were analyzed by immunocytochemistry. (E) Expression of CUDR was analyzed via RT-qPCR. All experiments were performed three times independently. ${ }^{*} \mathrm{P}<0.05$ vs. Day 0 group. All experiments were performed three independent times. HuMSCs, human umbilical cord mesenchymal stem cells; ALB, albumin; AFB, $\alpha$ fetoprotein; CYP3A4, cytochrome P450 3A4; HNF4 $\alpha$, hepatocyte nuclear factor $4 \alpha$; RT-qPCR, reverse transcription-quantitative PCR; CUDR, cancer upregulated drug resistant.

the same time, and the levels of hepatocyte-specific mRNAs such as ALB, AFP, CYP3A4 and HNF4 $\alpha$ were examined. It was shown that ALB, AFP, CYP3A4 and HNF4 $\alpha$ mRNA expression increased significantly during the period (Fig. 1C). The level of GAPDH was used to normalize the results, and results obtained from HuMSCs cultured in growth medium and primary hepatocytes were compared. Cells of day 0 were cultured in growth medium and cells of day 14,28 were cultured in differentiation medium. Hepatocytes were acquired from Beijing Beina Chuanglian Biotechnology Institute and were cultured in L-DMEM. Additionally, the protein levels of ALB, AFP and CYP3A4 were analyzed via immunofluorescence staining after 28 days of induction. These three proteins stained positive in HuMSC-derived hepatocyte-like cells. Additionally, undifferentiated cells, as the control group, were negative for AFP and CYP3A4, while the ALB staining was lower than hepatocyte-like cells (Fig. 1D). The level of CUDR was also examined during differentiation, and was gradually upregulated over time (Fig. 1E).
Overexpression of CUDR in hepatic differentiation. The overexpression of CUDR in hepatic differentiation was further probed by using a lentiviral construct. After confirming successful transfection by fluorescence imaging (data not shown), HuMSCs were selected by puromycin for at least 7 days. The results of RT-qPCR demonstrated that CUDR remained highly expressed during differentiation relative to the negative control (Fig. 2A). To explore the role of CUDR overexpression in hepatic differentiation, mRNAs of several hepatic markers were compared between HuMSCs transfected with CUDR, and HuMSCs transfected with the negative control, after 14 and 28 days of induction. As presented in Fig. 2C, the expression levels of ALB, CYP3A4, tyrosine aminotransferase (TAT), glucose-6-phosphatase (G6P) and $\alpha 1$-antitrypsin ( $\alpha 1 \mathrm{AT})$ mRNA in the CUDR overexpression group were higher than those in the control group $(\mathrm{P}<0.05)$ at day 14 and day 28. The expression of AFP in the CUDR overexpression group was lower than that in the control group at day 28 , indicating that these cells had a more mature status. 


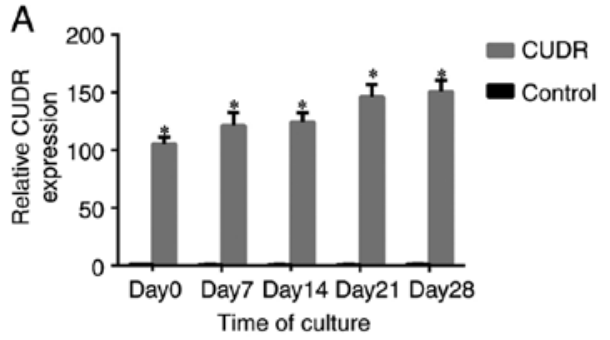

B
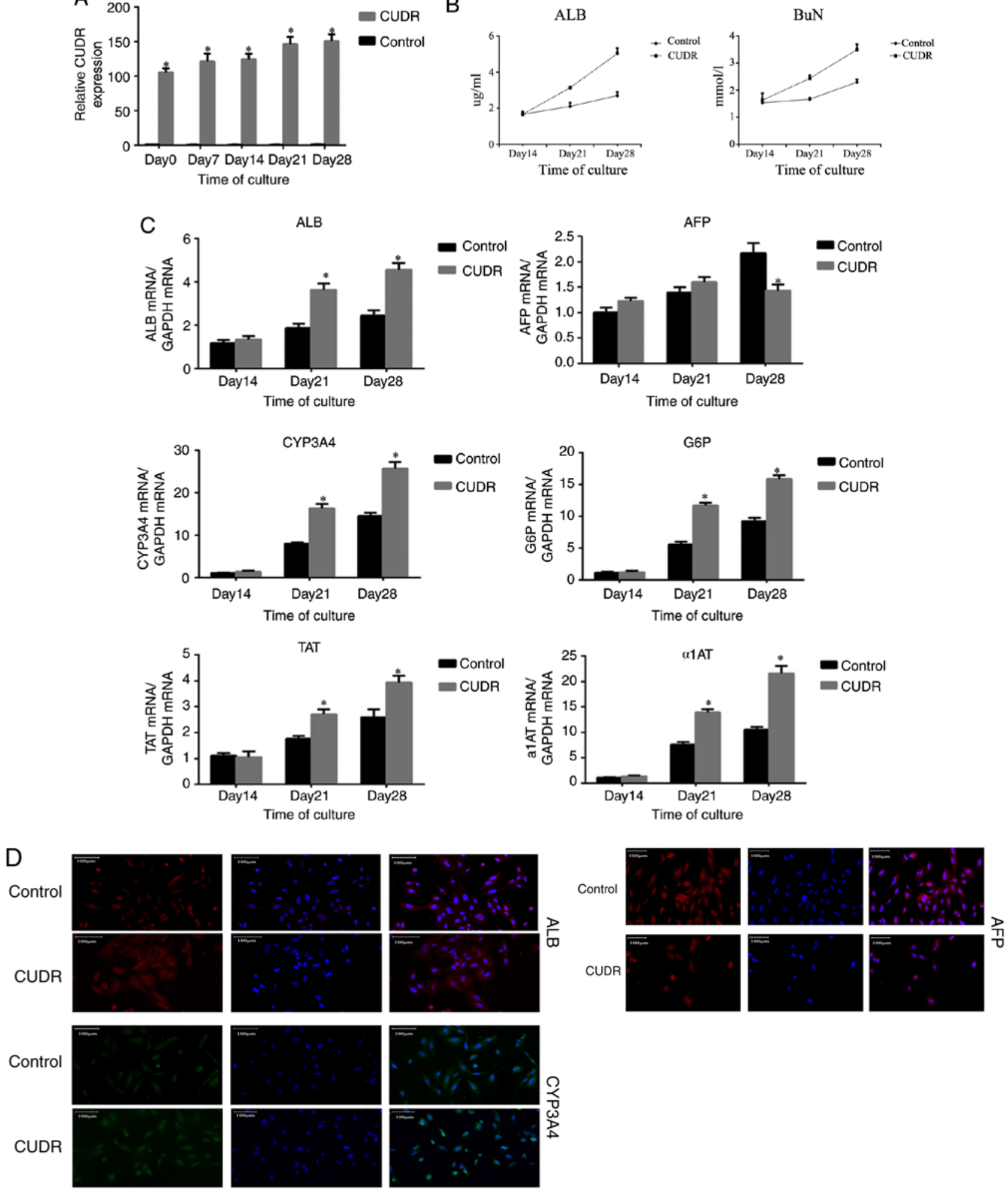

Figure 2. Effect of CUDR overexpression on hepatic differentiation. (A) RT-qPCR analysis of CUDR expression levels in HuMSCs transduced with lentiviruses containing either CUDR or negative control. (B) ALB and BuN concentration of the supernatant were analyzed by ELISA. (C) Hepatic differentiation was evaluated with reverse transcription-quantitative PCR analysis of ALB, AFP, CYP3A4, G6P, TAT and $\alpha 1$ AT. (D) Comparison of ALB, CYP3A4 and AFP protein between HuMSCs transfected with CUDR and cells cultured in medium containing cytokines via immunocytochemistry. ${ }^{*} \mathrm{P}<0.05$ vs. control group. All experiments were performed three independent times. HuMSCs, human umbilical cord mesenchymal stem cells; ALB, albumin; AFB, $\alpha$ fetoprotein; CYP3A4, cytochrome P450 3A4; CUDR, cancer up-regulated drug resistant; BuN, blood urea nitrogen; G6P, glucose-6-phosphatase; TAT, tyrosine aminotransferase; $\alpha 1 \mathrm{AT}, \alpha 1$-antitrypsin.

Furthermore, the ALB and BuN production at day 14 and day 28 showed a significant increase in CUDR (Fig. 2B). The expression of ALB, CYP3A4 and AFP proteins were also examined by immunostaining after 28 days of induction. Statistical analysis demonstrated that the HuMSCs transfected with CUDR showed higher expression of ALB and CYP3A4 

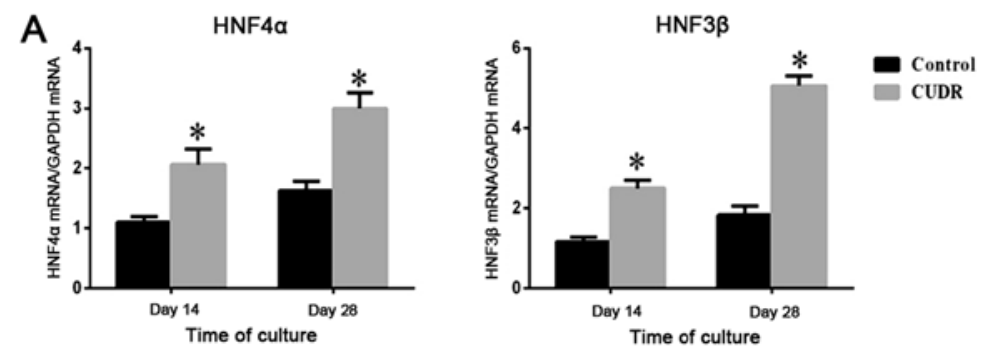

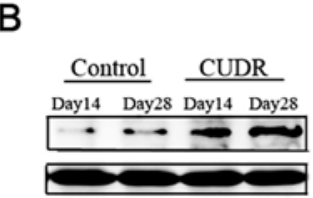

HNF4a

CEBPa

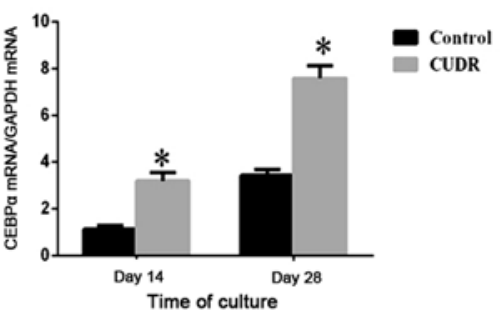

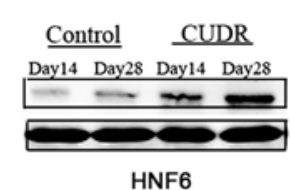

HNF6

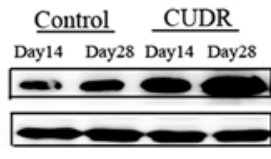

HNF3 $\beta$

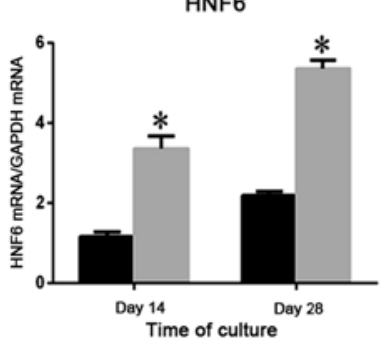

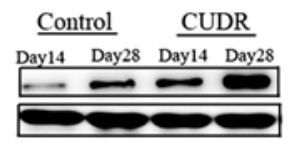

CEBPa

Figure 3. CUDR promotes hepatic differentiation by regulating liver-enriched factors. (A) mRNA of HNF4 $\alpha, \mathrm{HNF} 3 \beta$, HNF6 and CEBP $\alpha$ were analyzed by reverse transcription-quantitative PCR after 14 and 28 days of differentiation. (B) Protein levels of HNF4 $\alpha, H N F 3 \beta, H N F 6$ and CEBP $\alpha$ were analyzed by western blot analysis after 14 and 28 days of differentiation. ${ }^{*} \mathrm{P}<0.05$ vs. control group. All experiments were performed three independent times. HNF4 $\alpha$, hepatocyte nuclear factor $4 \alpha$; CUDR, cancer upregulated drug resistant; HNF3 $\beta$, hepatocyte nuclear factor $3 \beta$; HNF6, hepatocyte nuclear factor 6 ; CEBP $\alpha$, enhanced binding protein $\alpha$.
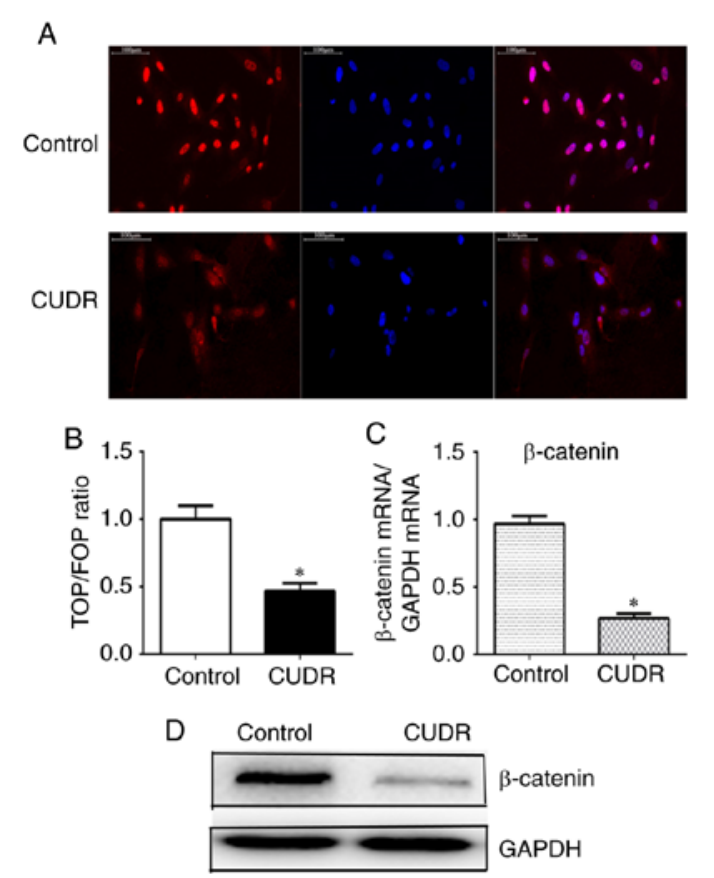

Figure 4. CUDR inhibits Wnt signaling activity by decreasing $\beta$-catenin cyto-nuclear translocation. (A) Immunocytochemistry staining for the localization of $\beta$-catenin in hepatocyte-like cells after differentiation when transfected with control or CUDR. (B) TOP/FOP assay showing the decreased activity of Wnt signaling in the hepatocyte-like cells with overexpression of CUDR. (C) RT-qPCR analysis showing CUDR transduction downregulated the mRNA expression of $\beta$-catenin. (D) Result of western blot analysis was in accordance with RT-qPCR. ${ }^{*} \mathrm{P}<0.05$ vs. control group. All experiments were performed three independent times. CUDR, cancer upregulated drug resistant; RT-qPCR, reverse transcription-quantitative PCR.

compared with the control group, while the expression of AFP protein level was lower compared with the control group, which was consistent with the results of RT-qPCR (Fig. 2D).
CUDR upregulates liver-enriched transcriptional factors. It has been demonstrated by several studies that the coordinated expression of liver-enriched transcriptional factors is required for the hepatic differentiation and biological functions of hepatocyte cells (18-20). It was shown in the control group that the expression levels of HNF4 $\alpha, \mathrm{HNF} 3 \beta, \mathrm{HNF} 6$ and CEBP $\alpha$ were low at days 14 and 28. After the overexpression of CUDR, these genes were expressed at a higher level on days 14 and 28 (Fig. 3A), which indicated their possible relationship with CUDR. Protein levels of liver-enriched factors were examined via western blotting and the results were in accordance with RT-qPCR analysis (Fig. 3B).

CUDR downregulates $\beta$-catenin. As is commonly known, nuclear translocation plays an important role in $\mathrm{Wnt} / \beta$-catenin signaling activation (21). To explore the mechanism underlying the relationship between CUDR and increased hepatic differentiation, the expression of $\beta$-catenin and the sub-cellular localization in HuMSCs were examined in the presence or absence of overexpression of CUDR. As shown in Fig. 4A, $\beta$-catenin was strongly expressed and mostly localized in the nucleus in the control group. However, following CUDR overexpression, the nuclear staining of $\beta$-catenin was markedly reduced. The TOP/FOP reporter assay revealed that the Wnt/ $\beta$-catenin signaling was significantly downregulated by CUDR overexpression (Fig. 4B). In line with these data, overexpression of CUDR significantly suppressed the expression of $\beta$-catenin mRNA and protein (Fig. 4C and D).

\section{Discussion}

It has been demonstrated that HuMSCs can differentiate into hepatocyte-like cells under certain conditions in vitro. HuMSCs have a major advantage over BMSCs and other sources of stem cells as they can be obtained non-invasively 
from umbilical cords. The fact that they are in vast abundance, free from ethical problems, cause a less pronounced immune response and offer the possibility to be cultured over a long time period in vitro make HuMSCs the ideal choice for the present study (6).

To generate hepatocyte-like cells, numerous researchers have engaged in finding simpler and more efficient procedures from various types of stem cells. In this study, HuMSCs were induced under hepatic differentiation media that included different cytokines and growth factors, which have been demonstrated to be important during the differentiation into hepatocyte-like cells. Then, hepatocyte-specific markers were examined to confirm the hepatic features of hepatocyte-like cells. Besides the changes of morphology that were examined by phase contrast microscopy, expression of hepatocyte-related genes such as AFP, ALB, CYP3A4 and HNF4 $\alpha$ were also confirmed via RT-qPCR analysis. Moreover, it was shown by immunofluorescence that ALB, AFP and CYP3A4 were expressed at a translation level.

The importance of specific lncRNAs participating in physiological and pathological processes, including the differentiation of various cells has been shown. For instance, Huang et al $(22,23)$ demonstrated that $\mathrm{H} 19$ could improve the osteogenic capacity of MSCs through the transforming growth factor- $\beta 1 /$ Smad family member $3 /$ histone deacetylase signaling pathway, while it inhibited adipocyte differentiation of BMSCs through epigenetic modulation of histone deacetylases. The lncRNA RAR related orphan receptor C contributes to SOX9 expression and chondrogenic differentiation of HuMSCs (24). Although CUDR is known to be involved in carcinogenesis at the transcriptional and post-transcriptional levels, CUDR is seldom reported to regulate hepatic differentiation (14). The results of the present study showed that the expression of CUDR gradually increased following the differentiation of HuMSCs. This study attempted to focus on the overexpression of CUDR in the hepatic differentiation of HuMSCs. In order to show this, the lentiviral transduction procedure was used to overexpress CUDR in HuMSCs, and the level of CUDR overexpression was examined using RT-qPCR. This revealed a continuous high expression of CUDR in transfected HuMSCs throughout the process of differentiation.

As expected, CUDR overexpression in HuMSCs resulted in a significant upregulation of several genes related to hepatic function. It was revealed by RT-qPCR that the expression levels of ALB, CYP3A4, TAT, G6P and $\alpha 1 \mathrm{AT}$ were higher than in the control group, whereas AFP was expressed on a lower level, indicating a more mature hepatocyte character (25). Furthermore, the secretion of ALB and the production of urea in the transduced cells were detected using ELISA. The results also demonstrated that CUDR overexpression induced a more functional hepatocyte-like cell. Additionally, the findings of immunofluorescence analysis of the ALB, AFP and CYP3A4 proteins were in accordance with the RT-qPCR results.

As commonly known, the coordinated expression of liver-enriched transcriptional factors is required for the hepatic differentiation and biological functions of hepatocytes $(19,20)$. In the present study, the liver-enriched factors mRNA HNF4 $\alpha$, HNF3 $\beta$, HNF6 and CEBP $\alpha$ began to increase after 14 days of induction as determined via RT-qPCR analysis. Overexpression of CUDR upregulated the liver-enriched factors compared with the control group, which indicated that CUDR may improve the function of hepatocyte-like cells via the regulation of liver-enriched factors.

Accumulating evidence indicates that the downregulation of Wnt/ $\beta$-catenin signaling contributes to the hepatic differentiation of MSCs (26-28). Several lncRNAs were found to participate in Wnt/ $\beta$-catenin signaling, including lnc liver regeneration 1 , lnc transcription factor 7 , and $\operatorname{lnc}$ $\beta$-Catm (29-31). The Wnt/ $\beta$-catenin signaling pathway was focused on in the present study as a possible mechanism for hepatic differentiation induced by CUDR overexpression. It was shown that the overexpression of CUDR prevented the translocation of $\beta$-catenin to the nucleus, and suppressed the activity of this pathway. Therefore, the enhanced hepatic differentiation of HuMSCs caused by CUDR may be linked to the downregulation of the $\mathrm{Wnt} / \beta$-catenin signaling pathway. However, the mechanism of how CUDR affects $\beta$-catenin level and sub-cellular localization needs further investigation.

To conclude, the findings of the present study indicated that CUDR may be a prominent factor in the hepatic differentiation of HuMSCs. This suggested that the overexpression of CUDR could serve as a valuable procedure to produce efficient and functional hepatocyte-like cells for transplantation. Therefore, this could be very useful in improving hepatic differentiation and providing a regenerative therapy for liver disease. However, further studies are required to clarify the particular mechanisms during hepatic differentiation.

\section{Acknowledgements}

Not applicable.

\section{Funding}

No funding was received.

\section{Availability of data and materials}

The datasets used and/or analyzed during the current study are available from the corresponding author on reasonable request.

\section{Authors' contributions}

FQ conceived and designed the experiments. YY performed the experiment and wrote the paper. ML, YS and JX performed the experiments and analyzed the data. All authors read and approved the final manuscript.

\section{Ethics approval and consent to participate}

Not applicable (HuMSCs were purchased from Beijing Beina Chuanglian Biotechnology Institute).

\section{Patient consent for publication}

Not applicable.

\section{Competing interests}

The authors declare that they have no competing interests. 


\section{References}

1. Meirelles Junior RF, Salvalaggio P, Rezende MB, Evangelista AS, Guardia BD, Matielo CE, Neves DB, Pandullo FL, Felga GE, Alves JA, et al: Liver transplantation: History, outcomes and perspectives. Einstein (Sao Paulo) 13: 149-152, 2015 (In English, Portuguese).

2. Baertschiger RM, Serre-Beinier V, Morel P, Bosco D, Peyrou M, Clément S, Sgroi A, Kaelin A, Buhler LH and Gonelle-Gispert C: Fibrogenic potential of human multipotent mesenchymal stromal cells in injured liver. PLoS One 4: e6657, 2009.

3. Lee KD, Kuo TK, Whang-Peng J, Chung YF, Lin CT, Chou SH, Chen JR, Chen YP and Lee OK: In vitro hepatic differentiation of human mesenchymal stem cells. Hepatology 40: 1275-1284, 2004

4. In 't Anker PS, Scherjon SA, Kleijburg-van der Keur C, de Groot-Swings GM, Claas FH, Fibbe WE and Kanhai HH: Isolation of mesenchymal stem cells of fetal or maternal origin from human placenta. Stem Cells 22: 1338-1345, 2004.

5. El-Tantawy WH and Haleem EN: Therapeutic effects of stem cell on hyperglycemia, hyperlipidemia, and oxidative stress in alloxan-treated rats. Mol Cell Biochem 391: 193-200, 2014.

6. Fan CG, Zhang QJ and Zhou JR: Therapeutic potentials of mesenchymal stem cells derived from human umbilical cord. Stem Cell Rev 7: 195-207, 2011.

7. Yu YB, Song Y, Chen Y, Zhang F and Qi FZ: Differentiation of umbilical cord mesenchymal stem cells into hepatocytes in comparison with bone marrow mesenchymal stem cells. Mol Med Rep 18: 2009-2016, 2018.

8. Ek M, Soderdahl T, Kuppers-Munther B, Edsbagge J, Andersson TB, Björquist P, Cotgreave I, Jernström B, Ingelman-Sundberg $M$ and Johansson I: Expression of drug metabolizing enzymes in hepatocyte-like cells derived from human embryonic stem cells. Biochem Pharmacol 74: 496-503, 2007.

9. Xu C, Zhang Y, Wang Q, Xu Z, Jiang J, Gao Y, Gao M, Kang J, Wu M, Xiong J, et al: Long non-coding RNA GAS5 controls human embryonic stem cell self-renewal by maintaining NODAL signalling. Nat Commun 7: 13287, 2016.

10. Wang L, Zhao Y, Bao X, Zhu X, Kwok YK, Sun K, Chen X, Huang Y, Jauch R, Esteban MA, et al: LncRNA Dum interacts with Dnmts to regulate Dppa2 expression during myogenic differentiation and muscle regeneration. Cell Res 25: 335-350, 2015.

11. Kalwa M, Hanzelmann S, Otto S, Kuo CC, Franzen J, Joussen S, Fernandez-Rebollo E, Rath B, Koch C, Hofmann A, et al: The lncRNA HOTAIR impacts on mesenchymal stem cells via triple helix formation. Nucleic Acids Res 44: 10631-10643, 2016.

12. Han Y, Yang YN, Yuan HH, Zhang TT, Sui H, Wei XL, Liu L, Huang P, Zhang WJ and Bai YX: UCA1, a long non-coding RNA up-regulated in colorectal cancer influences cell proliferation, apoptosis and cell cycle distribution. Pathology 46: 396-401, 2014.

13. Li T, Zheng Q, An J, Wu M, Li H, Gui X, Pu H and Lu D: SET1A Cooperates With CUDR to promote liver cancer growth and hepatocyte-like stem cell malignant transformation epigenetically. Mol Ther 24: 261-275, 2016

14. Gui X, Li H, Li T, Pu H and Lu D: Long Noncoding RNA CUDR Regulates HULC and $\beta$-catenin to govern human liver stem cell malignant differentiation. Mol Ther 23: 1843-1853, 2015.

15. Wang HS, Shyu JF, Shen WS, Hsu HC, Chi TC, Chen CP, Huang SW, Shyr YM, Tang KT and Chen TH: Transplantation of insulin-producing cells derived from umbilical cord stromal mesenchymal stem cells to treat NOD mice. Cell Transplant 20: 455-466, 2011

16. Ciciarello M, Zini R, Rossi L, Salvestrini V, Ferrari D, Manfredini R and Lemoli RM: Extracellular purines promote the differentiation of human bone marrow-derived mesenchymal stem cells to the osteogenic and adipogenic lineages. Stem Cells Dev 22: 1097-1111, 2013.
17. Livak KJ and Schmittgen TD: Analysis of relative gene expression data using real-time quantitative PCR and the 2(-Delta Delta C(T)) method. Methods 25: 402-408, 2001

18. Mihailovic M, Petrovic M and Bogojevic D: Correlation between acute phase-related expression of C/EBPbeta and transcriptional regulation of the haptoglobin gene during rat liver development. Gen Physiol Biophys 19: 317-321, 2000.

19. Costa RH, Kalinichenko VV, Holterman AX and Wang X Transcription factors in liver development, differentiation, and regeneration. Hepatology 38: 1331-1347, 2003.

20. Kyrmizi I, Hatzis P, Katrakili N, Tronche F, Gonzalez FJ and Talianidis I: Plasticity and expanding complexity of the hepatic transcription factor network during liver development. Genes Dev 20: 2293-2305, 2006.

21. MacDonald BT, Tamai K and He X: Wnt/beta-catenin signaling: Components, mechanisms, and diseases. Dev Cell 17: 9-26, 2009.

22. Huang Y, Zheng Y, Jia L and Li W: Long Noncoding RNA H19 promotes osteoblast differentiation Via TGF- $31 / \mathrm{Smad} 3 / \mathrm{HDAC}$ signaling pathway by deriving miR-675. Stem Cells 33: 3481-3492, 2015.

23. Huang Y, Zheng Y, Jin C, Li X, Jia L and Li W: Long Non-coding RNA H19 inhibits adipocyte differentiation of bone marrow mesenchymal stem cells through epigenetic modulation of histone deacetylases. Sci Rep 6: 28897, 2016

24. Barter MJ, Gomez R, Hyatt S, Cheung K, Skelton AJ, Xu Y, Clark IM and Young DA: The long non-coding RNA ROCR contributes to SOX9 expression and chondrogenic differentiation of human mesenchymal stem cells. Development 144: 4510-4521, 2017.

25. Deutsch HF: Chemistry and biology of alpha-fetoprotein. Adv Cancer Res 56: 253-312, 1991.

26. Ke Z, Zhou F, Wang L, Chen S, Liu F, Fan X, Tang F, Liu D and Zhao G: Down-regulation of Wnt signaling could promote bone marrow-derived mesenchymal stem cells to differentiate into hepatocytes. Biochem Biophys Res Commun 367: 342-348, 2008.

27. Shimomura T, Yoshida Y, Sakabe T, Ishii K, Gonda K, Murai R, Takubo K, Tsuchiya H, Hoshikawa Y, Kurimasa A, et al: Hepatic differentiation of human bone marrow-derived UE7T-13 cells: Effects of cytokines and CCN family gene expression. Hepatol Res 37: 1068-1079, 2007.

28. Yoshida Y, Shimomura T, Sakabe T, Ishii K, Gonda K, Matsuoka S, Watanabe Y, Takubo K, Tsuchiya H,Hoshikawa Y, et al: A role of Wnt/beta-catenin signals in hepatic fate specification of human umbilical cord blood-derived mesenchymal stem cells. Am J Physiol Gastrointest Liver Physiol 293: G1089-G1098, 2007.

29. Wang Y, He L, Du Y, Zhu P, Huang G, Luo J, Yan X, Ye B, Li C, Xia P, et al: The long noncoding RNA lncTCF7 promotes self-renewal of human liver cancer stem cells through activation of Wnt signaling. Cell Stem Cell 16: 413-425, 2015.

30. Zhu P, Wang Y, Huang G, Ye B, Liu B, Wu J, Du Y, He L and Fan Z: Inc- $\beta$-Catm elicits EZH2-dependent $\beta$-catenin stabilization and sustains liver CSC self-renewal. Nat Struct Mol Biol 23: 631-639, 2016.

31. Xu D, Yang F, Yuan JH, Zhang L, Bi HS, Zhou CC, Liu F, Wang $F$ and Sun SH: Long noncoding RNAs associated with liver regeneration 1 accelerates hepatocyte proliferation during liver regeneration by activating $\mathrm{Wnt} / \beta$-catenin signaling. Hepatology 58: 739-751, 2013.

his work is licensed under a Creative Commons Attribution-NonCommercial-NoDerivatives 4.0 International (CC BY-NC-ND 4.0) License. 